\title{
A Two-Sided Market Model of Optimal Price Structure for Instant Messenger
}

\author{
Jun Xu \\ Institute of Industrial Economics, Jinan University, Guangzhou 510632, China \\ Correspondence should be addressed to Jun Xu; crystaling@live.com
}

Received 11 October 2013; Accepted 12 November 2013

Academic Editor: Pu-yan Nie

Copyright (C) 2013 Jun Xu. This is an open access article distributed under the Creative Commons Attribution License, which permits unrestricted use, distribution, and reproduction in any medium, provided the original work is properly cited.

\begin{abstract}
Instant messenger (IM) is one of the most popular Internet applications all over the world. This paper examines the pricing problem of IM based on two-sided market model. IM serves as a two-sided platform, which gets both Internet users and advertisers on board. This paper concludes that IM operator adopts a heavily skewed price structure that favors IM users both under monopolistic case and under horizontal differentiated duopolistic case. When advertising revenue is large enough relatively to marginal cost for serving IM users, IM users can enjoy free service provided by IM operators. The competitive equilibrium of duopolistic case is not necessarily symmetric when advertisers choose singlehoming. Even in the symmetric equilibrium platform would rather deter all advertisers.
\end{abstract}

\section{Introduction}

Instant messenger (IM) is one of the most popular Internet applications all over the world. Especially in China, the leading IM Tencent QQ has more than 700 million active accounts while the latecomer WeChat has attracted 300 million users in just two years, though they belong to the same company, Tencent [1]. On the other side of Pacific Ocean, MSN messenger boasted around 330 million active monthly users in history [2], which is renamed as Windows Live Messenger and shifts users to Skype in 2013. Skype is not just only an instant messaging client but also provides voice-over-IP service which enables users to make Internet calls to mobiles and landlines. Inheriting user legacy of MSN messenger, Skype still holds 250 million monthly users in the context that Internet user's attention is greatly diverted to social network sites [3].

The reason for the popularity of IM is that people need to pay telecommunication carrier for their voice and SMS service, but they can enjoy free unlimited text and video communication offered by IM after fixed data plan payment to Internet service provider. IM operator's "Over the Top" (OTT) service poses a drastic innovation threat to traditional telecommunication carrier. However, a natural question emerges: how can IM providers sustain their business with free service for consumers? Some may argue that MSN messenger's provider Microsoft can cross-subsidize IM business by other profitable business such as Windows and Office. This argument cannot hold for independent provider such as Tencent and Skype before acquisition by Microsoft.

The most common practice for IM operator to retrieve revenue is to embed display ads on IM client software's user interface and sell ads slot to advertiser. If the IM can attract large amount of users and persuade them to stay enough time on the chatting box, IM users may see the ads and presumably click on the ads. Then IM operator can charge advertisers on the basis of cost per click (CPC). Thus, IM actually functions as a two-sided platform that connects IM users and advertisers.

The literature on two-sided markets is burgeoning in recent years. Two-sided markets are an intermediary agency, which enables two distinctive groups of agents to interact on this intermediary. Rochet and Tirole [4] analyzed the pricing decision of two-sided markets under different governance and different market structures, respectively. They found platform's pricing structure depends on the demand elasticity of two sides. Armstrong [5] considered the factors that influence platform pricing strategy in the presence of 
internetwork externality. He argued that the equilibrium price is determined by the magnitude of the cross-group externalities, whether fees are levied on a lump sum or pertransaction basis and whether agents join one platform or several platforms. Consolidating the model of Rochet and Tirole [4] and Armstrong [5], Rochet and Tirole [6] developed a unified framework for the two-sided market theory. By "corner" solution, Bolt and Tieman [7] proved that the side of the market which is sufficiently more elastic than the other side is kept to a minimum usage fee while the other side pays a relatively high usage fee. Hagiu [8] considered the case that producer provides diversified products to consumers through two-sided platform. In this case consumer prefers product variety while producers compete for consumers. $\mathrm{He}$ found that when consumer has strong preference for variety, the optimal price structure for the platform would extract more rents from consumer rather than producer. Weyl [9] took user heterogeneity into consideration. He found the platform could use insulating tariffs to avoid multi-equilibria and realize platform desired allocation structure. By relaxing product differentiation on both sides of platform, Armstrong and Wright [10] generalized the model of Armstrong [5] and proved "competitive bottleneck" may arise endogenously rather than exogenously as assumed by previous literature. The impact of exclusive dealing on equilibrium was also explored in their model.

The literature on advertising-supported media which is categorized by Evans [11] complements two-sided platform theory, although this line of literature developed earlier than two-sided markets. This line of literature shares some features with two-sided markets in that media can be regarded as two-sided platform. Nevertheless, the two-way positive intergroup network effects do not generally hold in the case of mass media. The crossnetwork externality that advertisers bring to audience is still in controversy although it is usually assumed negative. Anderson and Gabszewicz [12] surveyed this line of literature and argued that the fundamental tradeoff of media is balancing the positive externality which audience bring to advertisers and the possible negative externality which ads impose on audience. Anderson and Coate [13] modeled this tradeoff and compared the market provision equilibrium and social optima. Peitz and Valletti [14] adopted the same advertising demand function and compared the equilibrium of pay-TV and free-to-air model. Gal-Or and Dukes [15] employed Nash bargaining to model the transactional relation between two advertisers and two broadcasting stations. The highly concentrated product market in their model grants two producers who also advertise some monopsony power. Gabszewicz et al. [16] explicitly modeled the advertiser's choice on single-homing or multihoming. Multihoming enables advertiser to reach more audience but demands dual payments to both stations. Advertisers are vertical differentiated by the marginal profit derived from unit of audience. Advertisers who lie at the upper end of the market choose multihoming while those at the lower end only choose single-homing. Reisinger [17] endogenized overall usage time for watching TV and assumed usage time depends on the advertising level. He proved that platforms obtain positive margins in the advertising markets although there is
Bertrand competition for advertisers. Besides that, platform's profit increases in viewers nuisance cost of advertising.

Although theory of two-sided market is applied to various industries including mass media, IM rarely deserves the subject of literature. Faulhaber [18] pursued a case study of the merger between AOL and Time Warner and focused on the market power resulting from the merger in IM market. From the perspective of network externality, he proved in a market with increasing demand the market share of dominant firm increased at the expense of competitor's market share. Nevertheless, the intergroup network externality between IM users and advertisers was ignored in his study. Kim et al. [19] focused on the tying practice and showed that Microsoft can leverage its monopoly power in the operating system market to the instant messenger market through tying strategy. However, they did not touch the pricing problem.

Much of the study on two-sided markets takes banking card industry as research subject $[4,6,20]$, while the literature on advertising-supported media often takes traditional media such as TV broadcasting, newspaper, and magazine as example. To the best of our knowledge, the pricing problem of IM still lacks formal analysis. This is our writing motivation. In practice, IM is generally free of charge to consumers but sometimes IM charges some prices for specific service. For example, Skype charge consumers low rates for calls to mobiles and landlines (VoIP call). We try to find under which conditions IM is totally free and which circumstance consumers need to pay some money in our model.

The differences between our model and aforementioned two lines of literature are listed as follows.

Most of the literature on two-sided markets takes the total transaction volume between two sides as the output of the platform. We just add another dimension by incorporating user's online time duration into the two-sided markets model and redefine the output of IM platform that better illustrates the characteristic of behavioral patter of IM user.

Most of the literature on advertising-supported media neither ignores media's subscription charges to audience nor analyzes it in a discrete way. Our model consolidates their study in a continuous framework. Besides that the literature for TV broadcasting modeled users' switching behavior between different TV stations [16]. TV audience usually does not care about how much other audience watching the same station. However, IM users usually use only one IM and prefer the IM that is most popular among their friends. We assume single-homing and intragroup externality among IM users to better illustrate IM characteristics that are significantly different from TV audience.

The paper proceeds as follows. In Section 2 we present the monopolistic platform case. In Section 3 we present the duopolistic platform case. Section 4 contains conclusions and possible further extension.

\section{Monopolistic Platform}

The demand function by IM user is denoted as $n\left(p^{A}, a\right) . p^{A}$ is the one-shot registry fee charged by the platform, $a$ is the number of affiliated advertisers. Each advertiser is assumed to 
put unit of ads on IM platform, so the number of advertiser represents the amount of ads on the platform. $n$ is the number of IM users who register on the IM platform. We assume $\partial n / \partial p^{A}<0$ and $\partial n / \partial a<0$. That means higher registry fee and more advertisers frustrate more potential users to affiliate and vice versa.

Among those homogenous registered users, a representative user's online time duration is denoted as $t=t\left(n, p^{t}\right) \cdot p^{t}$ is the duration rate charged by the IM platform on the basis of how long IM users keep online while $n$ is the number of affiliated users. We assume that $\partial t / \partial n>0$ and $\partial t / \partial p^{t}<0$. These assumptions suggest that when the number of IM user is larger, the probability of user who signed in IM at any given time meeting with other online users is larger and the expected online time duration is longer. Higher time rate also forces IM to users spend less time online.

Advertiser's advertising demand function is $a=a(n t, r)$ where $n t$ is the total online time duration of all the IM users and $r$ is the advertising rate the platform charges from advertisers. For the sake of convenience, the advertising demand function rearranges to inverse functional form as $r(n t, a)$. It has the following property: $\partial r / \partial n t>0$ and $\partial r / \partial a<$ 0 . That means larger total online time duration raises the advertising rate while advertisers' demands less advertising slot under higher advertising rate.

Additional registered users require IM platform to increase the storage capacity to save users' profile and chatting $\log$ files. Such associated marginal cost is denoted by $c^{A}$. In the same way, we denote the marginal bandwidth cost for additional online user as $c^{t}$. Following the same specification as Gal-Or and Dukes [15], the marginal cost of advertising is set to zero since most of cost associated with production of advertising message is borne by advertising agency. So the profit function of IM platform can be formulated as follows

$$
\pi=\left(p^{A}-c^{A}\right) n+\left(p^{t}-c^{t}\right) n t+r(n t, a) a .
$$

Define $\varepsilon_{p^{A}}=-\left(\partial n / \partial p^{A}\right)\left(p^{A} / n\right)$ as elasticity of users registering with respect to price, $\varepsilon_{n \mid a}=(\partial n / \partial a)(a / n)$ as the elasticity of number of affiliated IM user with respect to number of advertisers, $\varepsilon_{p^{t}}=-\left(\partial t / \partial p^{t}\right)\left(p^{t} / t\right)$ as time elasticity with respect to time rate, $\varepsilon_{t \mid n}=(\partial t / \partial n)(n / t)$ as the time elasticity with respect to IM user number, $\varepsilon_{r}=-(\partial a / \partial r)(r / a)$ as price elasticity of advertising demand, and $\varepsilon_{r \mid n t}=(\partial r / \partial n t)(n t / r)$ as advertising demand elasticity with respect to the total time duration. From the assumption above, we can find that except for $\varepsilon_{r \mid n t}>0, \varepsilon_{t \mid n}>0$ the remaining elasticities are all negative. With these definitions, the following result can be proved.

Proposition 1. If nonnegative price constraint and proper second order sufficient condition holds, the optimal price structure of monopolistic platform consists of four cases according to different combination of parameter condition. The four cases are listed in Table 1.

Proof. See the appendix.

Proposition 1 states that if the marginal cost of platform is not very high, IM users will enjoy free communication service provided by IM operator. This conclusion contradicts the common sense that consumers are often hurt by high monopolistic price. Let us start from the first baseline case in second row and second column of Table 1 which IM platform charges positive prices to both sides of platform to explain such contradiction. Note that in single-sided platform without cross-group externalities, the optimal price structure would be

$$
\begin{aligned}
\frac{p^{A}-\left[c^{A}-\left(p^{t}-c^{t}\right)\left(1+\varepsilon_{t \mid n}\right) t\right]}{p^{A}}=\frac{1}{\varepsilon_{p^{A}}}, \\
\frac{p^{t}-c^{t}}{p^{t}}=\frac{1}{\varepsilon_{p^{t}}}, \\
\frac{r-0}{r}=\frac{1}{\varepsilon_{r}} .
\end{aligned}
$$

Expressions (2) imply that platform employs razor-blade business model [21]. The price of user registry is lower than its marginal cost by the factor $\left(p^{t}-c^{t}\right)\left(1+\varepsilon_{t \mid n}\right) t$. This factor can be decomposed into two parts: the first part $\left(p^{t}-c^{t}\right) t$ is the profit generated by additional user from his online time payments; the second part $\left(p^{t}-c^{t}\right) \varepsilon_{t \mid n} t$ is the profit from users' longer online time duration resulting from more users keeping online. However, duration rate charge is inflated to monopoly level to compensate the loss on lower registry price. On the other side of the market, expression (3) also indicates advertisers are levied monopolistic advertising rate although it looks a little odd. That is because advertising rate is assumed zero.

Comparing to single-sided platform, the price for twosided IM platform users is further adjusted downward by a factor $\varepsilon_{r \mid n t} \cdot a r / n t$. This term represents external benefits to platform which additional unit of users' total online time duration is attracted. To see this, note first that the platform makes profit ar/nt from extra unit of users' total online time. Second $\varepsilon_{r \mid n t}$ measures higher advertising rate that the platform can extract from advertisers without losing any advertisers.

On the other side of the platform, the advertising rate is adjusted upward by a factor $\left[\left(p^{A}-c^{A}\right)+\left(p^{t}-c^{t}+\right.\right.$ $\left.\left.(a r / n t) \varepsilon_{r \mid n t}\right)\left(1+\varepsilon_{t \mid n}\right) t\right] \varepsilon_{n \mid a}(n / a)$. This factor shares the same term $\left(p^{A}-c^{A}\right)+\left(p^{t}-c^{t}+(a r / n t) \varepsilon_{r \mid n t}\right)\left(1+\varepsilon_{t \mid n}\right) t$ with users' registry price. The reason for this common term is that raising registry price and increasing the number of advertisers both reduce the number of registered IM users. The term $\varepsilon_{n \mid a}$ measures the responsiveness of the number of affiliated IM users to advertisers while $n / a$ represents how many users per advertiser can reach IM platform. Certeris paribus, if advertiser can reach more users, IM platform can charge higher advertising rate without losing advertisers. Such rate is even higher than the single-sided monopoly market price level. This is because IM users are assumed to dislike ads. Therefore, IM platform increases the advertising rate to prevent too many ads from distracting IM users. In summary, the price structure of IM platform internalizes cross-group externality between users and advertisers by subsidizing users from advertising revenue. 
TABLE 1: The optimal price structure of monopolistic IM platform.

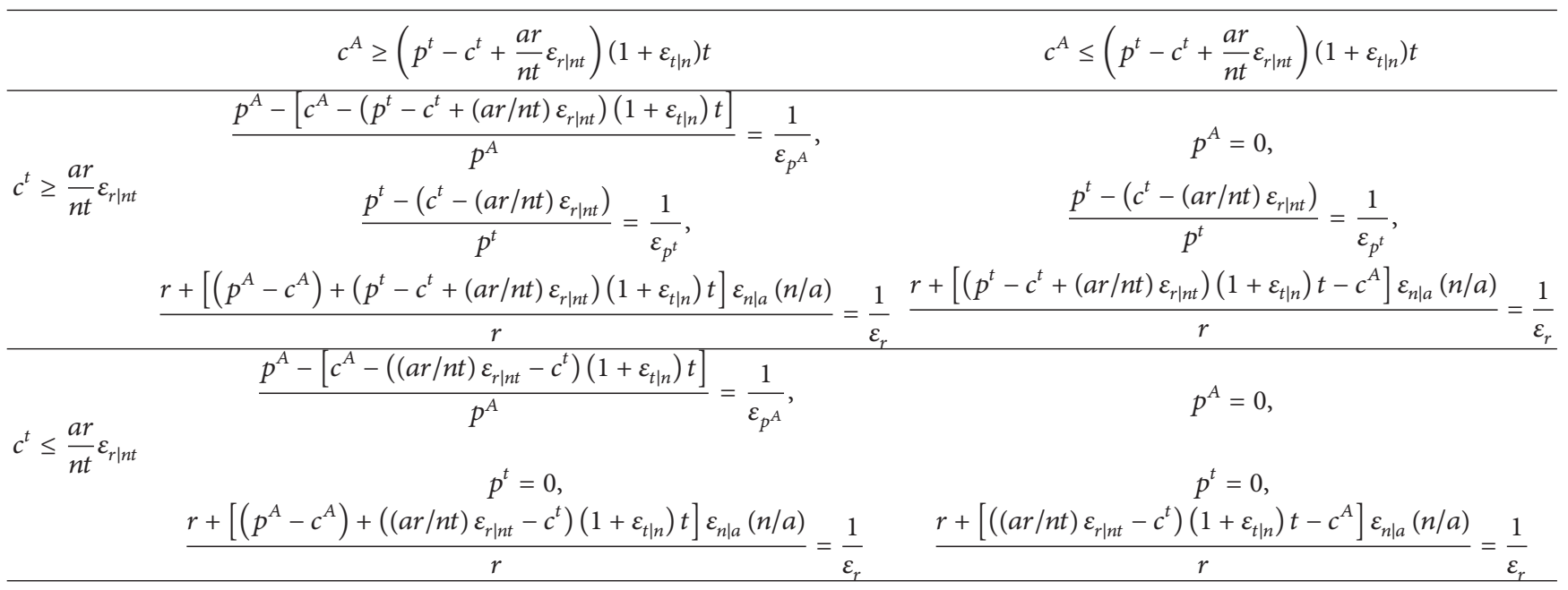

Note: the first row and first column of Table 1 list different combination of parameter region. The optimal price structure is summarized in cells located in the second and third rows of Table 1.

The remaining three cases, in which one or all of the prices are set to zero, are stemming from the aforementioned baseline case. The difference between them is that the marginal cost is so low that the nonnegative constraint is binding in the three remaining cases. Proposition 1 can help to explain why nearly all the IM client software is free to register but some of them (Skype) need to pay some money to make calls to mobile and landlines. That is because the marginal cost for IM users is so low comparing to the advertising revenue, which is generated by users' total online time. Users' registry price is double subsidized both under razor-blade business model and under two-sided market model. The reason for the positive charge of VoIP call by Skype is that Skype needs to pay the access charge fee to telecommunication carrier. That causes the marginal online cost to be substantially higher than that of other IM without VoIP function. Thus, positive price is applied to Skype VoIP call though it is still lower than actual marginal cost due to subsidies from advertising revenue.

\section{Duopolistic Platforms}

Referring to the model specification of horizontal differentiated duopolist outlined by Armstrong [5] and Armstrong and Wright [10], two IM platforms are located in the two ends of linear market, respectively. Users were uniformly distributed on this linear market, which indicates users have different preference for two IM platforms.

Every user can only affiliate to one IM platform and the two platforms are not compatible. User located in $n_{1}$ derives utility of $U_{1}=v_{0}+\alpha n_{1}-p_{1}^{A}-\tau n_{1}-\omega a_{1}$ when he/she registered on platform 1. $v_{0}$ is user's intrinsic utility when he/she affiliates to any platform. $\alpha$ is the strength of intragroup network externality or the incremental utility generated by an additional user. $n_{1}$ can also represent the share of how many users affiliated with platform $1 . p_{1}^{A}$ is the registry fee charged by platform 1 to users. $\tau$ is user's virtual transport cost which also can represent the difference between two platforms. $a_{1}$ is the number of advertisers who put ads on platform 1 , and $\omega$ is IM users' nuisance cost for ads. In the same manner, the utility user derived from registering on IM platform 2 is denoted by $U_{2}=v_{0}+\alpha n_{2}-p_{2}^{A}-\tau n_{2}-\omega a_{2}$ and $n_{1}+n_{2}=1$. According to Hotelling model, the number of users who affiliate to each IM platforms is listed, respectively:

$$
\begin{aligned}
& n_{1}=\frac{1}{2}+\frac{p_{2}^{A}-p_{1}^{A}+\omega\left(a_{2}-a_{1}\right)}{2(\tau-\alpha)}, \\
& n_{2}=\frac{1}{2}+\frac{p_{1}^{A}-p_{2}^{A}+\omega\left(a_{1}-a_{2}\right)}{2(\tau-\alpha)} .
\end{aligned}
$$

We make assumption $\alpha-\tau<0$ which means the difference between two IM platforms is larger than the intragroup network externality. Therefore, neither platform can cover the whole market. Expression (4) indicates that when the registry fee of platform 1 is lower and the advertisers who connect to platform 1 are fewer, more users will register on platform 1 , and vice versa.

The online time duration of user on platform 1 is assumed as $t_{1}\left(n_{1}, p_{1}^{t}\right)=\beta n_{1}-p_{1}^{t} . n_{1}$ is the number of IM users who affiliate with platform $1 . \beta>0$ indicates the more users register on platform 1 , the longer the representative user keeps online. $p_{1}^{t}$ is the duration rate charged by platform 1 on the basis of time length. Users will spend less time if the duration rate is higher. In the same manner the online time duration of users on platform 2 is $t_{2}\left(n_{2}, p_{2}^{t}\right)=\beta n_{2}-p_{2}^{t}$.

If advertiser places ads on platform $i$, the total profit he/she derives from users' total online time duration is denoted by $\theta n_{i} t_{i}$. And advertiser needs to pay advertising rate $r_{i}$ to platform $i$. So the net surplus which advertiser obtains from placing ads on platform $i$ can be formulated as $\theta n_{i} t_{i}-r_{i}$. If advertisers choose single-homing, they only need to pay single advertising rate to single platform, but his/her ads can only be viewed by single platform's IM users. If advertisers choose multihoming, their ads can reach all the IM users at expense of double advertising rate. Since two platforms are 
homogeneous to advertisers, advertisers only care about net surplus attributed to advertising on platforms. Advertisers will choose the alternatives with highest net surplus from multihoming, single-homing on platform 1, single-homing on platform 2, and no affiliation to any platform. When advertisers choose multihoming, their net surplus must be larger than the other alternatives. So we must have $\theta\left(n_{1} t_{1}+\right.$ $\left.\theta n_{2} t_{2}\right)-r_{1}-r_{2} \geq \theta n_{1} t_{1}-r_{1}, \theta\left(n_{1} t_{1}+\theta n_{2} t_{2}\right)-r_{1}-r_{2} \geq \theta n_{2} t_{2}-$ $r_{2}$ and $\theta\left(n_{1} t_{1}+\theta n_{2} t_{2}\right)-r_{1}-r_{2} \geq 0$. Rearranging and combining these expressions we get $\theta n_{1} t_{1} \geq r_{1}, \theta n_{2} t_{2} \geq r_{2}$. Following the same manner, when advertisers choose single-homing on platform 1, we get $\theta n_{1} t_{1} \geq r_{1}, \theta n_{2} t_{2} \leq r_{2}$. When advertisers choose single-homing on platform 2 , we get $\theta n_{1} t_{1} \leq r_{1}$, $\theta n_{2} t_{2} \geq r_{2}$. When advertisers do not affiliate to any platform, we get $\theta n_{1} t_{1} \leq r_{1}, \theta n_{2} t_{2} \leq r_{2}$. The homing choice of advertisers divides advertiser's parameter space into four regions. Then we will discuss the optimal pricing decision under four cases.

3.1. Advertiser Multihoming $\left(\theta n_{1} t_{1} \geq r_{1}, \theta n_{2} t_{2} \geq r_{2}\right)$. The number of advertisers is unitized to be one. The profit function of platform 1 can be formulated as follows

$$
\begin{aligned}
& \pi_{1}=\left(p_{1}^{A}-c^{A}\right) n_{1}+\left(p_{1}^{t}-c^{t}\right) n_{1} t_{1}+r_{1} \\
& \text { s.t. } \quad \theta n_{1} t_{1} \geq r_{1}, \quad \theta n_{2} t_{2} \geq r_{2} .
\end{aligned}
$$

The profit of platform 2 can be formulated in the same manner. The following proposition presents the optimal price structure of two platforms under symmetric equilibrium. Because the equilibrium is symmetric, the subscript number of price pair which indicates platform 1 or 2 is suppressed for short.

Proposition 2. With nonnegative price constraint and proper second order sufficient condition holding, the optimal price structure of duopolistic platform under symmetric equilibrium consists of four cases according to different combination of parameter condition. The four cases are listed in Table 2.

Proof. See the appendix.

It still makes sense to compare the first baseline case in which IM platform charges positive price with singlesided platform case. When there is no cross-group externality between IM users and advertisers, the optimal price structure under Hotelling model is

$$
\begin{gathered}
p^{A}=c^{A}+\tau-\alpha-\frac{\left((1 / 2) \beta-c^{t}\right)\left((3 / 2) \beta-c^{t}\right)}{4}, \\
p^{t}=\frac{\beta+2 c^{t}}{4}, \\
r=\frac{\theta\left(\beta-2 c^{t}\right)}{8} .
\end{gathered}
$$

It is obvious that the IM platform still employs the razorblade business model, which subsidizes registry fee with online duration profit. Comparing expressions (6) to the cell in the second row and second column of Table 2, the prices for IM users are adjusted downward by advertisers' unit profit from users' total online time duration. The reasoning is the same as in the monopolistic case. Besides that, all of advertisers' profit is extracted by two platforms to subsidize users. They are in the same circumstance with monopolistic case. That is because they choose multihoming, their bargaining power does not change even when IM platforms are competing with each other.

It is interesting to compare the equilibrium outcome between monopolistic platform and duopolistic case with advertisers multihoming. Unlike monopolistic case, the virtual transport cost raises the registry fee while intra-group externality reduces it. Since infinite virtual transport cost is equivalent to monopolistic case, moderate virtual transport cost in duopolistic case actually cuts the registry fee. Intragroup externality among users is more likely to lead to highly concentrated market. Thus, duopolistic platforms lower their registry fee to avoid their customers being distracted to each other. However, their registry fee offsets each other. In duopolistic equilibrium, IM users are allocated equally to two IM platforms due to symmetric registry fee.

Proposition 2 shows that in the symmetric equilibrium the nonnegative constraint on the pricing of the platform results in four different pricing configurations. The difference of Propositions 1 and 2 is that the prerequisites for zero price are determined by additional factors. These factors include virtue transport $\operatorname{cost} \tau$, intra-group externality $\alpha$, and responsiveness of user online time duration to the number of affiliated users $\beta$. From the first row of Table 2, virtual transport cost associated with marginal cost of users' registry narrows the likelihood of zero registry fee, while intra-group externality together with double subsidy from razor-blade and two-sided market business makes IM users more likely to enjoy free registry service. From the first column of Table 2, responsiveness of user online time duration to the number of affiliated users makes it less likely for IM platform charges zero price for user's online time duration, ceteris paribus. This is because when users spend longer time online due to more affiliated other users, they may derive higher utility from online communication. Platform is privileged to demand more user surplus by a higher time duration rate.

3.2. Advertisers Single-Homing on Platform $1\left(\theta n_{1} t_{1} \geq r_{1}\right.$, $\left.\theta n_{2} t_{2} \leq r_{2}\right)$. The profit function of platform 1 is the same as in the multihoming case. The profit function of platform 2 can be formulated as follows:

$$
\begin{aligned}
& \pi_{2}=\left(p_{2}^{A}-c^{A}\right) n_{2}+\left(p_{2}^{t}-c^{t}\right) n_{2} t_{2} \\
& \text { s.t. } \quad p_{2}^{A} \geq 0, \quad p_{2}^{t} \geq 0 .
\end{aligned}
$$

The following proposition depicts the equilibrium of single-homing. 
TABLE 2: The optimal price structure of duopolistic IM platforms when advertisers choose multi-homing.

\begin{tabular}{cc}
\hline$c^{A}+\tau>\alpha+\frac{\left((1 / 2) \beta-c^{t}+\theta\right)\left((3 / 2) \beta-c^{t}+\theta\right)}{4}$ & $c^{A}+\tau<\alpha+\frac{\left((1 / 2) \beta-c^{t}+\theta\right)\left((3 / 2) \beta-c^{t}+\theta\right)}{4}$ \\
\hline $\begin{array}{l}p^{A}=c^{A}+\tau-\alpha-\frac{\left((1 / 2) \beta-c^{t}+\theta\right)\left((3 / 2) \beta-c^{t}+\theta\right)}{4}, 2 \theta \\
p^{t}=\frac{\beta+2 c^{t}-2 \theta}{4},\end{array}$ & $p^{t}=\frac{\beta+2 c^{t}-2 \theta}{4}$, \\
$r=\frac{\theta\left(\beta-2 c^{t}+2 \theta\right)}{8}$ & $r=\frac{\theta\left(\beta-2 c^{t}+2 \theta\right)}{8}$ \\
\hline$c^{A}+\tau>\alpha+\beta\left(\theta-c^{t}\right)$ & $c^{A}+\tau<\alpha+\beta\left(\theta-c^{t}\right)$ \\
$p^{A}=c^{A}+\tau-\alpha-\beta\left(\theta-c^{t}\right)$, & $p^{A}=0$, \\
$p^{t}=0$, & $p^{t}=0$, \\
$r=\frac{\beta \theta}{4}$ & $r=\frac{\beta \theta}{4}$
\end{tabular}

Note: the first and third rows and the first column of Table 1 list different combination of parameter region. The optimal price structure is summarized in the cells located in the second and fourth rows of Table 2 .

Proposition 3. Under proper second order sufficient condition, the optimal price for users of two platform is listed as follows:

$$
\begin{gathered}
p_{1}^{A}=c^{A}+\tau-\alpha-\frac{2}{3} \frac{\left(\beta n_{1}-c^{t}+\theta\right)\left(3 \beta n_{1}-c^{t}+\theta\right)}{4} \\
-\frac{1}{3} \frac{\left(\beta n_{2}-c^{t}\right)\left(3 \beta n_{2}-c^{t}\right)}{4}, \\
p_{1}^{t}=\frac{\beta n_{1}+c^{t}-\theta}{2}, \\
p_{2}^{A}=c^{A}+\tau-\alpha-\frac{1}{3} \frac{\left(\beta n_{1}-c^{t}+\theta\right)\left(3 \beta n_{1}-c^{t}+\theta\right)}{4} \\
-\frac{2}{3} \frac{\left(\beta n_{2}-c^{t}\right)\left(3 \beta n_{2}-c^{t}\right)}{4}, \\
p_{2}^{t}=\frac{\beta n_{2}+c^{t}}{2}, \\
r_{2}=+\infty .
\end{gathered}
$$

The number of users affiliated with platform 1 is

$$
n_{1}=\frac{1}{2}+\frac{4 \omega-\theta\left(2 \beta+\theta-c^{t}\right)}{2\left(3 \beta^{2}-4 \beta c^{t}+2 \beta \theta-12 \tau+12 \alpha\right)} .
$$

Proof. See the appendix.

From expression (9), we can find that the pricing of platform 2 follows the traditional principle of monopoly price. Since there is no ad on platform 2 , the subsidy for online time duration rate from advertising profit is not feasible. Therefore, the online duration rate is strictly positive. Nevertheless, registry fee is subsidized by razor-blade business model, which makes it is possible to below zero.

Expressions (8) and (9) also show the registry fee of platforms 1 and 2 depends on virtual transport cost, the intra-group externality, and profits derived from online time duration. Registry fee decreases when the virtual transport cost is smaller and the intra-group externality is larger. It is noteworthy that although there is no ad in platform 2 , the profit parameter $\theta$ which advertiser derives from per unit of online time duration enters into the registry fee of platform 2 . That reflects the strategic effect of two platforms.

Expression (10) indicates the number of users who affiliated to platform 1 depends on the parameter regions. In different parameter regions, the market share of platform can be either above $1 / 2$ or below it. It is the same for platform 2 . The reason for such result is that advertisers are assumed homogeneous. So as long as the advertising rate is lower than the profit derived from advertising on IM platform, all the advertisers will connect to the platform and vice versa. As platform cannot control the number of affiliated advertisers precisely, the market share of users for two platforms is hardly symmetric. Since the registry fee and duration rate all depend on market share of users, the competitive equilibria are not necessarily symmetric. Only if $4 \omega=\theta\left(2 \beta+\theta-c^{t}\right)$, then the market share of affiliated users belonging to two platforms both equals to $1 / 2$. In this circumstance, the duration rate of platform 1 is lower than that of platform 2 because of subsidy from advertising profit. In the meantime, singlehoming advertisers also make the registry fee of platform 1 lower than that of platform 2 .

3.3. Advertisers Single-Homing on Platform $2\left(\theta n_{1} t_{1} \leq\right.$ $\left.r_{1}, \theta n_{2} t_{2} \geq r_{2}\right)$. This case is symmetric with previous case. Therefore, the conclusion is also the same as that in Proposition 3. 
3.4. Advertiser Nonaffiliation $\left(\theta n_{1} t_{1} \leq r_{1}, \theta n_{2} t_{2} \leq r_{2}\right)$. In this case, two platforms charge a rather high advertising rate in order to deter all the advertisers. Therefore, two platforms degenerate into single-sided market. After simple derivation, the prices charged to users under symmetric equilibrium are given in expression (6).

After four cases are analyzed, the next step is to make a comparison of these four cases. Denote the profit of the platforms under multihoming case and nonaffiliating as $\pi^{M}$ and $\pi^{N}$. It is easy to verify

$$
\begin{gathered}
\pi^{M}=\frac{1}{16}\left[8(\tau-\alpha)-\beta^{2}+2 \beta c^{t}-2 \beta \theta\right], \\
\pi^{N}=\frac{1}{16}\left[8(\tau-\alpha)-\beta^{2}+2 \beta c^{t}\right] .
\end{gathered}
$$

Obviously $\pi^{M}<\pi^{N}$. Comparing to the symmetric equilibrium under multihoming, platforms obtain more profits by deterring all advertisers. This is resulting from the subsidy structure of platform. No matter under multihoming or nonaffiliation, platforms will subsidize registry fee by online time duration profit motivated by razor-blade business model. Although the online time duration profit is different under multihoming case and nonaffiliation case, two platforms engage in Hotelling style symmetric competition, and two symmetric platforms obtain the same online time duration profit under multihoming case. This result also holds under no-affiliation case. Nevertheless, platform not only subsidizes registry fee by online time duration profit but also subsidizes it with extra profit resulting from the change of user online time duration. Such part of extra profit is different under multihoming and nonaffiliation. It is $\left(p^{t}-c^{t}+\theta\right) \beta n_{i}$ under multihoming and $\left(p^{t}-c^{t}\right) \beta n_{i}$ under nonaffiliation. The reason for such difference is that under multihoming case platform will subsidize online time duration rate by the profit extracted from advertisers. However, this cannot happen under nonaffiliation case. Therefore, in the symmetric equilibrium, multihoming platforms need to subsidize more to users than nonaffiliation platforms, leading to less profit for itself.

When advertisers choose single-homing on platform 1 , it is easy to derive the profit of platform $1 \pi_{1}$ and platform $2 \pi_{2}$, respectively:

$$
\begin{gathered}
\pi_{1}=\frac{-\beta^{2} n_{1}+\beta c^{t}-\beta \theta+4 \tau-4 \alpha}{2} n_{1}^{2}, \\
\pi_{2}=\frac{-\beta^{2} n_{2}+\beta c^{t}+4 \tau-4 \alpha}{2} n_{2}^{2} .
\end{gathered}
$$

It is obvious that the profit of two platforms depends on their affiliated users. As mentioned earlier, the market share of users depends on relevant parameter regions. The relative size of profits belonging to two platforms also depends on that parameter regions. No explicit conclusion can be drawn except for one special case when $n_{1}=n_{2}$. It can be proved that $\pi_{1}=\pi^{M}$ and $\pi_{2}=\pi^{N}$. Therefore, IM platform would rather charge infinite high advertising rate to deter all advertisers.

In complement to previous conclusions, the following proposition can be summarized as follows.
Proposition 4. In the symmetric equilibrium of horizontal differentiated duopolistic platform, if all the advertisers derive the same profit from each unit of online time duration and two platforms attract equal number of users, platforms will choose a rather high advertising rate to deter all the advertisers.

\section{Conclusion}

The popularity of IM among Internet users all over the world represents the rise of digital media. The reason for the popularity of IM is that it provided a rather cheap communication alternative to people scattered in different corner of the earth. However, IM rarely deserves the subject of economic literature, especially for its pricing decision. Motivated by this gap, this paper investigates the optimal price structure of IM platform. Being different from literature on two-sided markets and advertising-supported media, this paper redefine the output of IM platform by adding another time dimension to two-sided market model. Besides that, this paper assumes intra-group externality among IM users, which better illustrates the behavioral pattern of IM users.

The general conclusion of this paper is that under nonnegative price constraint, IM operator adopts a heavily skewed price structure that favors IM users. In concrete terms, firstly under monopolistic case, the IM platform double-subsidizes users' price motivated by the razorblade and two-sided market business model. Advertisers are charged above-monopolistic-level advertising rate. When advertising revenue is large enough relatively to marginal cost for serving IM users, IM user can enjoy free service provided by IM operators. Secondly, under duopolistic case when advertisers choose multihoming, the equilibrium is almost the same as monopolistic case; besides that the virtual transport cost and responsiveness of user online time duration to the number of affiliated users could raise users' price. Thirdly, when advertisers choose single-homing, the IM platform with no ads charges strictly positive online time duration rates, but strategy effect leads platform also to subsidize registry fee with advertising revenue. Fourthly, if two platforms attract equal number of users, platforms would choose a rather high advertising rate to deter all the advertisers.

Our conclusion helps to explain why IM operator charges rather low even zero price for IM users. Especially we clarify under which conditions IM users could enjoy free communication service provided by IM. Although we take IM as context of our research, our conclusion also sheds some light on the pricing problem of social network sites (SNS), whose users share similar behavioral pattern to that of IM users. Our result is still consistent with the free policy provided for users by SNS operators in reality.

Next, this study can extend to heterogeneous user case. We assume user homogeneity in terms of online time duration. In fact, different users may spend different length of time online. Moreover, some users who spend longer online period have higher willing to pay for online virtual goods. Platform may utilize such user heterogeneity to gain more revenue. That is effective method for Tencent, which is the biggest IM 
operator in China. Such kind of price discrimination deserves our further research.

\section{Appendix}

Proof of Proposition 1. The IM platform makes choice of $p^{A}$, $p^{t}$, and $r$ to maximize the total profit with the nonnegative pricing constraint. The Kuhn-Tucker first order necessary condition for profit maximization is listed as follows:

$$
\begin{aligned}
\frac{\partial \pi}{\partial p^{A}}=n+\left[p^{A}-c^{A}+\left(p^{t}-c^{t}+a \frac{\partial r}{\partial n t}\right)\left(t+n \frac{\partial t}{\partial n}\right)\right] \frac{\partial n}{\partial p^{A}} \leq 0 \\
p^{A} \geq 0, \quad \frac{\partial \pi}{\partial p^{A}} p^{A}=0 \\
\frac{\partial \pi}{\partial p^{t}}=n t+n\left(p^{t}-c^{t}+a \frac{\partial r}{\partial n t}\right) \frac{\partial t}{\partial p^{t}} \leq 0, \\
\frac{\partial \pi}{\partial a}=\left[\left(p^{A}-c^{A}\right)+\left(p^{t}-c^{t}+a \frac{\partial r}{\partial p^{t}} p^{t}=0,\right.\right. \\
+r+a \frac{\partial r}{\partial a} \leq 0, \quad r \geq 0, \frac{\partial \pi}{\partial a} r=0 .
\end{aligned}
$$

According to complementary slackness, when $p^{A}>0$ and $p^{t}>0, \partial \pi / \partial p^{A}$, and $\partial \pi / \partial p^{t}$ in (A.1) are set to zero. Rearranging these two expressions, we have

$$
\begin{gathered}
\frac{p^{A}-\left[c^{A}-\left(p^{t}-c^{t}+(a r / n t) \varepsilon_{r \mid n t}\right)\left(1+\varepsilon_{t \mid n}\right) t\right]}{p^{A}}=\frac{1}{\varepsilon_{p^{A}}} \\
\frac{p^{t}-\left(c^{t}-(a r / n t) \varepsilon_{r \mid n t}\right)}{p^{t}}=\frac{1}{\varepsilon_{p^{t}}} .
\end{gathered}
$$

$c^{t} \geq(\operatorname{ar} / n t) \varepsilon_{r \mid n t}$ and $c^{A} \geq\left(p^{t}-c^{t}+(a r / n t) \varepsilon_{r \mid n t}\right)\left(1+\varepsilon_{t \mid n}\right) t$ are necessary for $p^{A}>0$, and $p^{t}>0$ together with (A.2). If (A.2) holds, $\partial \pi / \partial r=0$ can be rearranged to be

$$
\begin{aligned}
& \frac{r+\left[\left(p^{A}-c^{A}\right)+\left(p^{t}-c^{t}+(a r / n t) \varepsilon_{r \mid n t}\right)\left(1+\varepsilon_{t \mid n}\right) t\right] \varepsilon_{n \mid a}(n / a)}{r} \\
& =\frac{1}{\varepsilon_{r}} .
\end{aligned}
$$

Because $\varepsilon_{r \mid n t}>0, \varepsilon_{t \mid n}>0, \varepsilon_{n \mid a}<0$ the second term in the numerator in (A.3) is negative. So that $r>0$ and it never equals zero. Expressions (A.2) and (A.3) constitute the cell in second row and second column of Table 1 .

When $p^{A}=0$ and $p^{t}>0, \partial \pi / \partial p^{A}$ needs to be negative and $\partial \pi / \partial p^{t}$ in (A.1) is set to zero. Expression $\partial \pi / \partial p^{t}=0$ implies

$$
\frac{p^{t}-\left(c^{t}-(a r / n t) \varepsilon_{r \mid n t}\right)}{p^{t}}=\frac{1}{\varepsilon_{p^{t}}} .
$$

Expression (A.4) requires $c^{t} \geq(\operatorname{ar} / n t) \varepsilon_{r \mid n t}$ for $p^{t}>0$ to hold. Inequation $\partial \pi / \partial p^{A}>0$ together with $p^{A}=0$ requires $\left[c^{A}-\left(p^{t}-c^{t}+a(\partial r / \partial n t)\right)(t+n(\partial t / \partial n))\right]\left(\partial n / \partial p^{A}\right)>n$. Nevertheless, $c^{A} \leq\left(p^{t}-c^{t}+(a r / n t) \varepsilon_{r \mid n t}\right)\left(1+\varepsilon_{t \mid n}\right) t$ is sufficient for this inequation to hold. If $p^{A}=0$ and expression (A.4) holds, $\partial \pi / \partial r=0$ is rearranged to be

$$
\frac{r+\left[\left(p^{t}-c^{t}+(a r / n t) \varepsilon_{r \mid n t}\right)\left(1+\varepsilon_{t \mid n}\right) t-c^{A}\right] \varepsilon_{n \mid a}(n / a)}{r}=\frac{1}{\varepsilon_{r}} .
$$

Because $\varepsilon_{r \mid n t}>0, \varepsilon_{t \mid n}>0, \varepsilon_{n \mid a}<0$ the second term in the numerator in (A.5) is negative. So that $r>0$ and it never equals zero. $p^{A}=0,(\mathrm{~A} .4)$ and (A.5) constitute the cell in second row and third column of Table 1 . The proof for the rest part of Proposition 1 follows the same reasoning and can be omitted for convenience. Besides that, the Hessian matrix of the maximizing problem evaluated at the solution given above should be seminegative definite to guarantee the sufficient condition for maximal. Proof of Proposition 1 is complete.

Proof of Proposition 2. Because all the advertisers place ads on platform 1, the profit function of platform 1 is linear in advertising rate. The optimal choice of platform is to set the advertising rate at the highest level that can keep all the advertisers choosing multihoming. Then $r_{1}=\theta n_{1} t_{1}$. The target function of platform under nonnegative price constraints is

$$
\begin{aligned}
& \pi_{1}=\left(p_{1}^{A}-c^{A}\right) n_{1}+\left(p_{1}^{t}-c^{t}\right) n_{1} t_{1}+\theta n_{1} t_{1} \\
& \text { s.t. } \quad p_{1}^{A} \geq 0, \quad p_{1}^{t} \geq 0 .
\end{aligned}
$$

The Kuhn-Tucker first order necessary condition for profit maximization is listed as follows:

$$
\begin{gathered}
\frac{\partial \pi_{1}}{\partial p_{1}^{A}}=\left[p_{1}^{A}-c^{A}+\left(p_{1}^{t}-c^{t}+\theta\right)\left(2 \beta n_{1}-p_{1}^{t}\right)\right] \frac{\partial n_{1}}{\partial p_{1}^{A}} \\
+n_{1} \leq 0, \quad p_{1}^{A} \geq 0, \quad \frac{\partial \pi_{1}}{\partial p_{1}^{A}} p_{1}^{A}=0, \\
\frac{\partial \pi_{1}}{\partial p_{1}^{t}}=n_{1}\left[\beta n_{1}-p_{1}^{t}-p_{1}^{t}+c^{t}-\theta\right] \leq 0, \\
p_{1}^{t} \geq 0, \quad \frac{\partial \pi_{1}}{\partial p_{1}^{t}} p_{1}^{t}=0 .
\end{gathered}
$$

According to complementary slackness, when $p_{1}^{A}>0$, and $p_{1}^{t}>0$, then $\left(\partial \pi_{1} / \partial p_{1}^{A}\right)=0,\left(\partial \pi_{1} / \partial p_{1}^{t}\right)=0$. Setting $\partial \pi_{1} / \partial p_{1}^{A}$, and $\partial \pi_{1} / \partial p_{1}^{t}$ in (A.7) equal to zero, we get

$$
\begin{gathered}
{\left[p_{1}^{A}-c^{A}+\left(p_{1}^{t}-c^{t}+\theta\right)\left(\beta n_{1}-p_{1}^{t}\right)\right] \frac{\partial n_{1}}{\partial p_{1}^{A}}} \\
+n_{1}\left[1+\left(p_{1}^{t}-c^{t}+\theta\right) \beta \frac{\partial n_{1}}{\partial p_{1}^{A}}\right]=0, \\
p_{1}^{t}=\frac{\beta n_{1}+c^{t}-\theta}{2} .
\end{gathered}
$$


Substitute (A.9) into (A.8) we have

$$
p_{1}^{A}-c^{A}+\frac{\left(\beta n_{1}-c^{t}+\theta\right)\left(3 \beta n_{1}-c^{t}+\theta\right)}{4}=2(\tau-\alpha) n_{1} .
$$

In the same manner we also have

$$
\begin{gathered}
p_{2}^{t}=\frac{\beta n_{2}+c^{t}-\theta}{2}, \\
p_{2}^{A}-c^{A}+\frac{\left(\beta n_{2}-c^{t}+\theta\right)\left(3 \beta n_{2}-c^{t}+\theta\right)}{4}=2(\tau-\alpha) n_{2} .
\end{gathered}
$$

Taking (A.10) minus (A.12) we get

$$
\begin{aligned}
& p_{1}^{A}-p_{2}^{A} \\
& +\frac{\left(\beta n_{1}-c^{t}+\theta\right)\left(3 \beta n_{1}-c^{t}+\theta\right)-\left(\beta n_{2}-c^{t}+\theta\right)\left(3 \beta n_{2}-c^{t}+\theta\right)}{4} \\
& \quad=2(\tau-\alpha)\left(n_{1}-n_{2}\right) .
\end{aligned}
$$

Because in multihoming case the two platforms have equal number of advertisers $\left(a_{1}=a_{2}\right)$, the difference of IM users between two platforms reduces to $n_{1}-n_{2}=\left(p_{2}^{A}-p_{1}^{A}\right) /(\tau-\alpha)$. Expression (A.13) at last can be reformulated as

$$
3 \beta^{2} \frac{p_{2}^{A}-p_{1}^{A}}{\tau-\alpha}-4 c^{t} \beta \frac{p_{2}^{A}-p_{1}^{A}}{\tau-\alpha}+4 \theta \beta \frac{p_{2}^{A}-p_{1}^{A}}{\tau-\alpha}=12\left(p_{2}^{A}-p_{1}^{A}\right) .
$$

Expression (A.14) always holds only if $p_{1}^{A}=p_{2}^{A}$. So we get $n_{1}=n_{2}=1 / 2$ and $p_{1}^{t}=p_{2}^{t}=p^{t}$. Substitute these results into (A.9), (A.10), (A.11), and (A.12). We have the cell in second row and second column of Table 2 . Condition $c^{A}+\tau>\alpha+\left(\left((1 / 2) \beta-c^{t}+\theta\right)\left((3 / 2) \beta-c^{t}+\theta\right) / 4\right)$ and $\beta+2 c^{t}>2 \theta$ is necessary for $p_{1}^{A}>0$, and $p_{1}^{t}>0$ together with $p^{A}=c^{A}+\tau-\alpha-\left(\left((1 / 2) \beta-c^{t}+\theta\right)\left((3 / 2) \beta-c^{t}+\theta\right) / 4\right)$ and $p^{t}=\left(\beta+2 c^{t}-2 \theta\right) / 4$. $r$ can be derived accordingly. If $c^{A}+\tau<\alpha+\left(\left((1 / 2) \beta-c^{t}+\theta\right)\left((3 / 2) \beta-c^{t}+\theta\right) / 4\right)$ and $\beta+2 c^{t}>2 \theta$, then $p^{A}=c^{A}+\tau-\alpha-(((1 / 2) \beta-$ $\left.\left.c^{t}+\theta\right)\left((3 / 2) \beta-c^{t}+\theta\right) / 4\right)<0$. The nonnegative constraint of $p^{A}$ is binding. Therefore, we have the cell in second row and third column of Table 2 . If $\beta+2 c^{t}<2 \theta$, then $p^{t}=$ $\left(\beta+2 c^{t}-2 \theta\right) / 4<0$, and the nonnegative constraint of $p^{t}$ is binding. So $p^{t}$ is set to zero in this circumstance. Taking this condition as given, the symmetric solution of $p_{1}^{A}, p_{2}^{A}$ satisfies $p^{A}=c^{A}+\tau-\alpha-\beta\left(\theta-c^{t}\right)$. When $c^{A}+\tau>\alpha+\beta\left(\theta-c^{t}\right)$, $p^{A}>0$ is consistent with nonnegative constraint. Then we have the cell in fourth row and second column of Table 2. When $c^{A}+\tau<\alpha+\beta\left(\theta-c^{t}\right), p^{A}<0$ is inconsistent with nonnegative constraint. That constraint must be binding so we have the cell in fourth row and third column of Table 2. Besides that, the Hessian matrix of the maximizing problem evaluated at the solution given above should be seminegative definite to guarantee the sufficient condition for maximal. Proof of Proposition 2 is complete.
Proof of Proposition 3. The decision of platform 1 is the same as the previous case that all advertisers choose multihoming. The first order condition is the same as multihoming case for platform 1. Platform 2 chooses a rather high advertising rate to deter all the advertisers. Since there are no ads on platform 2, its target function can be formulated as

$$
\pi_{2}=\left(p_{2}^{A}-c^{A}\right) n_{2}+\left(p_{2}^{t}-c^{t}\right) n_{2} t_{2}
$$

According to the first order condition for maximization we have

$$
\begin{gathered}
\frac{\partial \pi_{2}}{\partial p_{2}^{A}}=\left[p_{2}^{A}-c^{A}+\left(p_{2}^{t}-c^{t}\right)\left(2 \beta n_{2}-p_{2}^{t}\right)\right] \frac{\partial n_{2}}{\partial p_{2}^{A}}+n_{2}=0 \\
p_{2}^{t}=\frac{\beta n_{2}+c^{t}}{2}
\end{gathered}
$$

From (A.16) we get

$$
p_{2}^{A}-c^{A}+\frac{\left(\beta n_{2}-c^{t}\right)\left(3 \beta n_{2}-c^{t}\right)}{4}=2(\tau-\alpha) n_{2} .
$$

When all the advertisers choose single-homing on platform 1 , the number of affiliated users on platform 1 and platform 2 is listed, respectively:

$$
\begin{aligned}
& n_{1}=\frac{1}{2}+\frac{p_{2}^{A}-p_{1}^{A}-\omega}{2(\tau-\alpha)}, \\
& n_{2}=\frac{1}{2}+\frac{p_{1}^{A}-p_{2}^{A}+\omega}{2(\tau-\alpha)} .
\end{aligned}
$$

Substituting $n_{1}, n_{2}$ from (A.18) into (A.10) and (A.17) and then solving the simultaneous equations, we can get first expression in (8) and (9). The first expression in (8) minus the first expression in (9) together with (A.18) implies expression (10). Besides that, the Hessian matrix of the maximizing problem evaluated at the solution given above should be seminegative definite to guarantee the sufficient condition for maximal. Proof of Proposition 3 is complete.

\section{Acknowledgments}

This work was supported by the National Social Science Foundation of China (09\&ZD021). Sincere thanks to two anonymous reviewers for their valuable suggestions for revision.

\section{References}

[1] A. C. Beattie, "Marketers eye WeChat," Advertising Age, vol. 84, no. 8, pp. 20-20, 2013.

[2] A. Spiegel, "Microsoft to buy skype for $\$ 8.5$ billion: how to not screw it up," Forbes.com, pp. 27-37.

[3] T. I. M. Woodall, "Connecting the world," Strad, vol. 124, no. 1473, pp. 56-60, 2013.

[4] J.-C. Rochet and J. Tirole, "Platform competition in two-sided markets," Journal of the European Economic Association, vol. 1, no. 4, pp. 990-1029, 2003. 
[5] M. Armstrong, "Competition in two-sided markets," RAND Journal of Economics, vol. 37, no. 3, pp. 668-691, 2006.

[6] J.-C. Rochet and J. Tirole, "Two-sided markets: a progress report," RAND Journal of Economics, vol. 37, no. 3, pp. 645-667, 2006.

[7] W. Bolt and A. F. Tieman, "Heavily skewed pricing in two-sided markets," International Journal of Industrial Organization, vol. 26, no. 5, pp. 1250-1255, 2008.

[8] A. Hagiu, "Two-sided platforms: product variety and pricing structures," Journal of Economics \& Management Strategy, vol. 18, no. 4, pp. 1011-1043, 2009.

[9] E. G. Weyl, "A price theory of multi-sided platforms," American Economic Review, vol. 100, no. 3, pp. 1642-1672, 2010.

[10] M. Armstrong and J. Wright, "Two-sided markets, competitive bottlenecks and exclusive contracts," Economic Theory, vol. 32, no. 2, pp. 353-380, 2007.

[11] D. S. Evans, "The antitrust economics of multi-sided platform markets," Yale Journal on Regulation, vol. 20, no. 2, pp. 325-381, 2003.

[12] S. P. Anderson and J. J. Gabszewicz, "The media and advertising: a tale of two-sided markets," in Handbook of Cultural Economics, V. Ginsburgh and D. Throsby, Eds., vol. 1, Elsevier Science, Amsterdam, The Netherlands, 2005.

[13] S. P. Anderson and S. Coate, "Market provision of broadcasting: a welfare analysis," Review of Economic Studies, vol. 72, no. 3, pp. 947-972, 2005.

[14] M. Peitz and T. M. Valletti, "Content and advertising in the media: pay-tv versus free-to-air," International Journal of Industrial Organization, vol. 26, no. 4, pp. 949-965, 2008.

[15] E. Gal-Or and A. Dukes, "Minimum differentiation in commercial media markets," Journal of Economics and Management Strategy, vol. 12, no. 3, pp. 291-325, 2003.

[16] J. Gabszewicz, D. Laussel, and N. Sonnac, "TV broadcasting competition and advertising," CORE Discussion Papers 2000006, 2001.

[17] M. Reisinger, "Platform competition for advertisers and users in media markets," International Journal of Industrial Organization, vol. 32, no. 2, pp. 243-252, 2012.

[18] G. Faulhaber, "Network effects and merger analysis: instant messaging and the AOL-time warner case," Telecommunications Policy, vol. 26, no. 5-6, pp. 311-333, 2002.

[19] J. Kim, S. H. Bang, and S. Hwang, "Anti-competitiveness of instant messenger tying by microsoft," Hitotsubashi Journal of Economics, vol. 52, no. 2, pp. 185-198, 2011.

[20] J.-C. Rochet and J. Wright, "Credit card interchange fees," Journal of Banking and Finance, vol. 24, no. 8, pp. 1788-1797, 2010.

[21] R. Gil and W. R. Hartmann, "Empirical analysis of metering price discrimination: evidence from concession sales at movie theaters," Marketing Science, vol. 28, no. 6, pp. 1046-1062, 2009. 


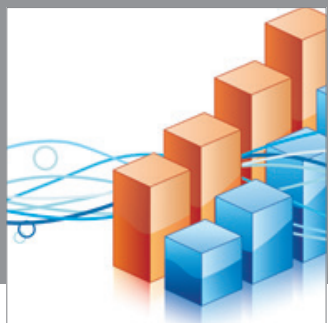

Advances in

Operations Research

mansans

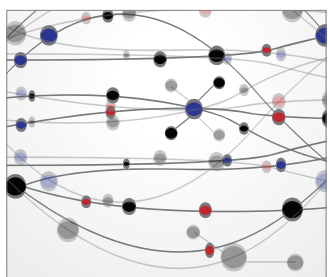

The Scientific World Journal
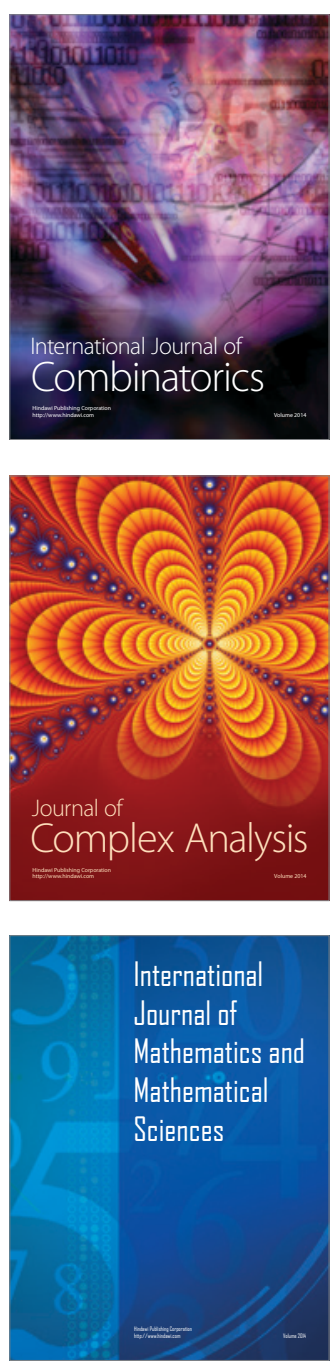
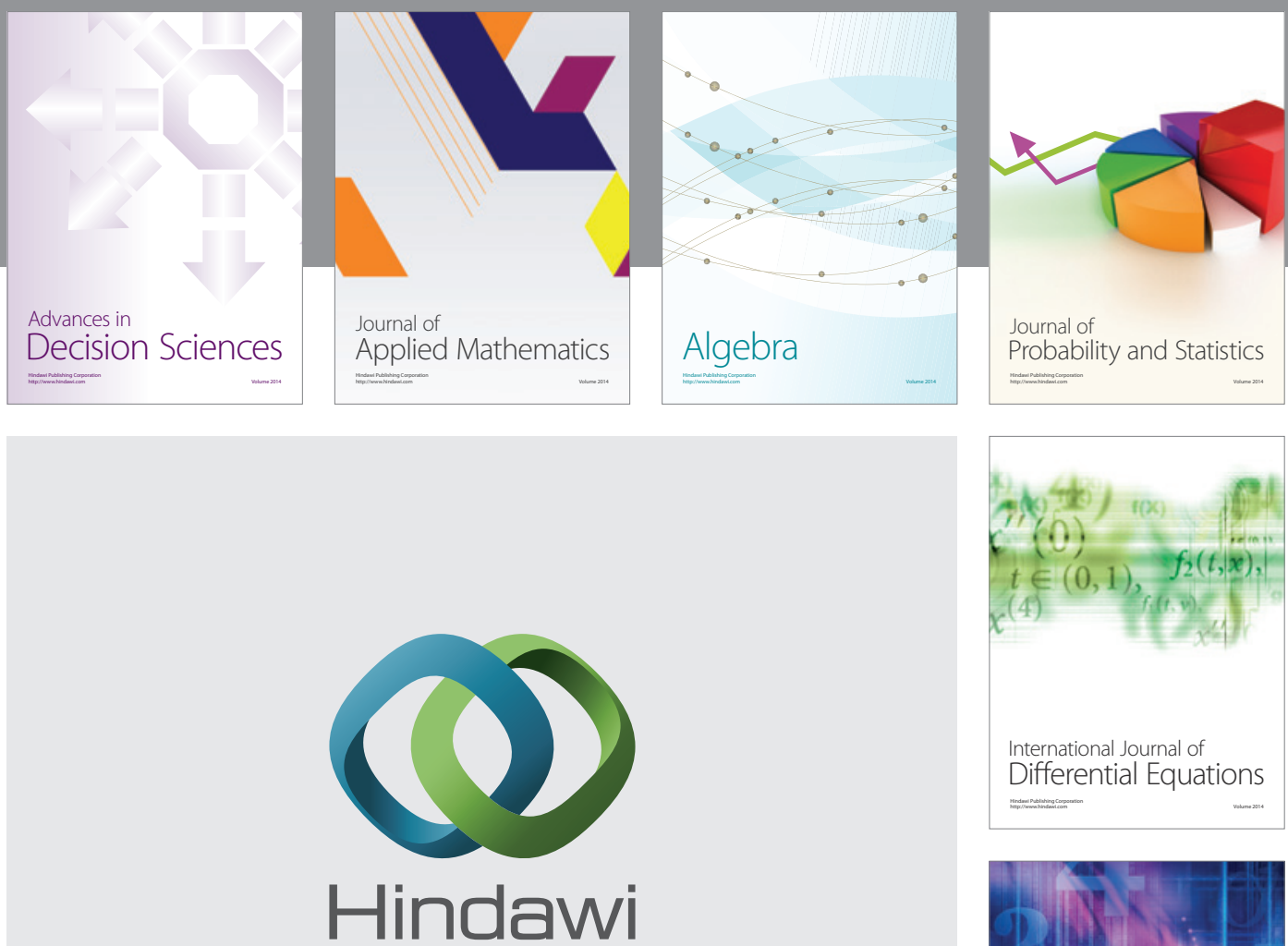

Submit your manuscripts at http://www.hindawi.com
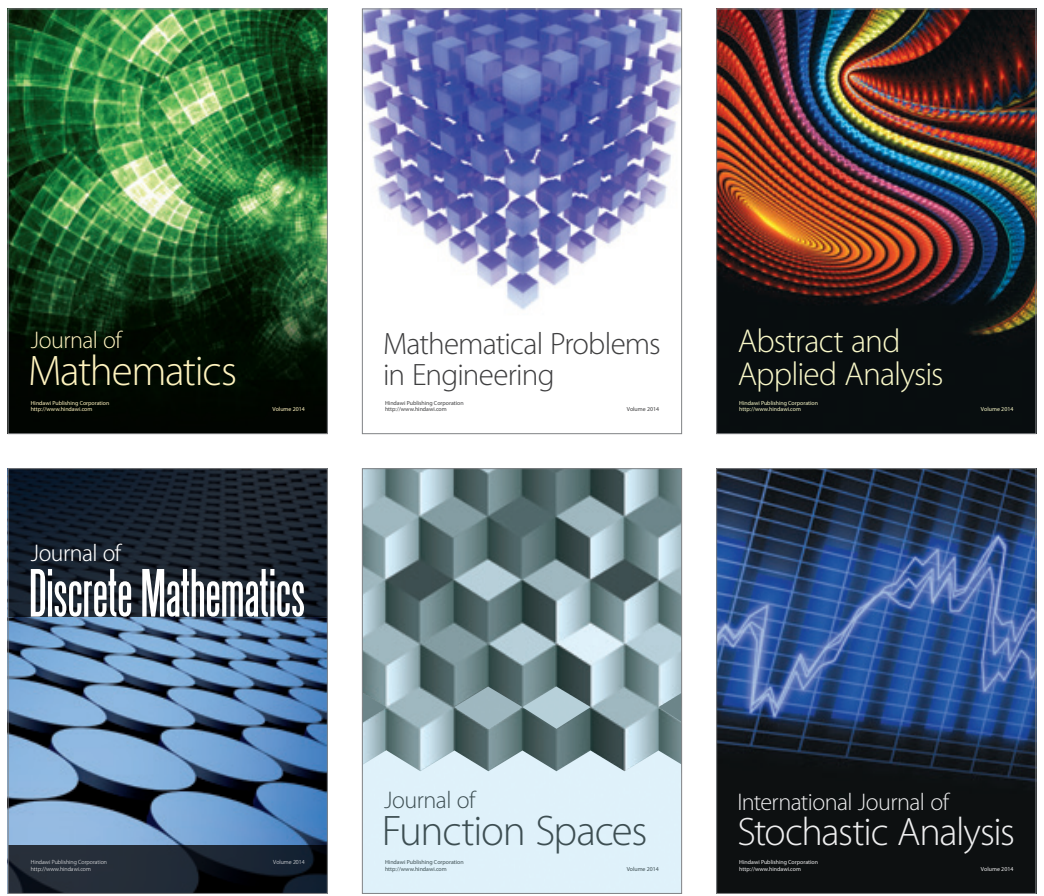

Journal of

Function Spaces

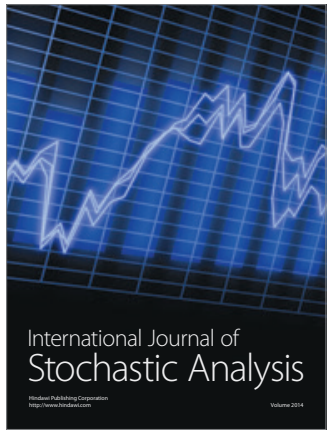

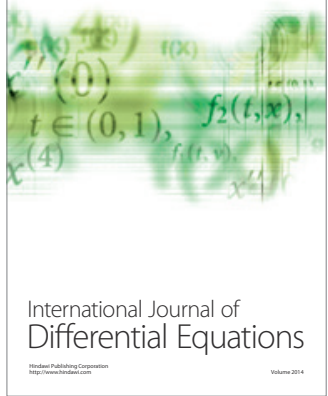
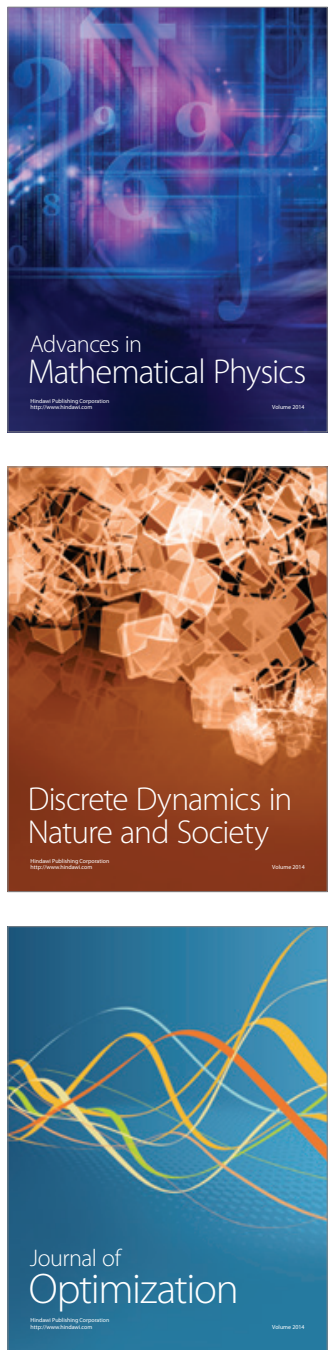